\title{
Propiedades psicométricas del index of spouse abuse en una muestra de varones españoles
}

\author{
psychometric properties of the index of spouse abuse in a spanish male sample
}

\author{
Pablo Santos-Iglesias \\ Juan Carlos Sierra \\ Pablo Vallejo-Medina \\ Universidad de Granada, España
}

(Rec:06 de julio de 2012 / Acep: 19 de octubre de 2012)

\begin{abstract}
Resumen
El objetivo del presente estudio fue analizar algunas propiedades psicométricas de la versión española del Index of Spouse Abuse en una muestra de varones españoles, ya que no existen instrumentos validados en España en esta población. Participaron 598 varones con edades comprendidas entre 18 y 77 años $(M$ $=40.20 ; D T=13.71)$ seleccionados de la población general mediante muestreo incidental. Los resultados mostraron una estructura de 30 ítems agrupados en tres subescalas: Abuso no físico, Control de conductas y Abuso físico. Los valores de fiabilidad oscilaron entre .60 y .81. Mostró buena validez convergente con las puntuaciones de la Sexual Assertiveness Scale. Se encontró que las conductas de abuso más frecuentes son de abuso no físico y control de conductas, siendo el abuso físico menos frecuente. Se discuten los resultados obtenidos y sus implicaciones para la evaluación de la violencia de pareja en varones.

Palabras clave: Index of Spouse Abuse, propiedades psicométricas, varones, violencia de pareja.
\end{abstract}

\begin{abstract}
The aim of the presente study was to analyze some psychometric properties of the Index of Spouse Abuse in a Spanish male sample, because there are no adapted instruments in Spain for this population. Participants were 598 males with ages ranging from 18 to 77 years old $(M=40.20 ; S D=13.71)$, and they were selected from general population through a convenience sampling procedure. Results showed a structure made up of 30 items clustered into three subscales: Nonphysical abuse, Behavior controlling, and Physical abuse. Reliability values ranged from .60 to .81 . It showed good convergent validity with scores on the Sexual Assertiveness Scale. Further, it was found that nonphysical abuse and behavior controlling were the most frequent form of abuse, while physical abuse was less frequent. Results and their implications for the assessment of partner abuse are discussed.

Key words: Index of Spouse Abuse, psychometric properties, males, partner violence
\end{abstract}

\section{Introducción}

La violencia de pareja es un problema que tiene consecuencias no sólo para las personas que lo sufren, sino también para la sociedad (Loinaz, Echeburua, y Ullate, 2012; Loinaz, Ortiz-Tallo y Ferragut, 2012; Novo, Fariña, Seijo, y Arce, 2012). Tradicionalmente su estudio se centró en la evaluación de las mujeres que sufrían abusos por parte de sus parejas varones (Kapur y Windish, 2011; Rodríguez-Franco et al., 2012), pero en la actualidad estudios realizados con muestras comunitarias han indicado que los varones son víctimas de violencia de pareja con la misma frecuencia e incluso mayor que las mujeres (Archer, 2002; Goldenson, Spidel, Greaves, y Dutton, 2009; Holtzworth-Munroe, 2005; Ibaceta, 2011; Póo y Vizcarra, 2011; Randle y Graham, 2011; Saldivia y Vizcarra, 2012). Además, se ha encontrado que los hombres víctimas de violencia de pareja también sufren consecuencias tanto físicas como psicológicas. Así,

Agradecimientos: Este estudio forma parte del proyecto SEJ2007-61824, concedido por el Ministerio de Ciencia e Innovación de España al segundo autor.

Correspondencia: Juan Carlos Sierra. Facultad de Psicología. Campus de Cartuja, s/n. 18011. E-mail: jcsierra@ugr.es 
en su reciente revisión, Randle y Graham (2011) señalan que pueden mostrar síntomas de estrés postraumático, trastorno de estrés postraumático, depresión e ideación suicida. Por tanto, la violencia de pareja de las mujeres hacia los varones es un problema que no puede ser ignorado y al que hay que dedicar esfuerzos y recursos (Carney, Buttell, y Dutton, 2007).

Uno de estos esfuerzos tiene que dirigirse al desarrollo y validación de instrumentos de evaluación de la violencia de pareja, ya que la mayoría de ellos han sido desarrollados para evaluar a las mujeres, siendo más escasos aquellos que evalúan la violencia hacia los hombres (Hays y Emelianchik, 2009; Thompson, Basile, Hertz, y Sitterle, 2006). Por ejemplo, el Index of Spouse Abuse (ISA; Hudson y McIntosh, 1981) es uno de los instrumentos de evaluación de la violencia de pareja más empleado (Feldhaus et al., 1997). Fue desarrollado para evaluar la violencia de pareja en la mujer. En su versión original, está compuesto por 30 ítems que evalúan la frecuencia de abuso tanto físico como no físico por parte de la pareja. Cada uno de esos 30 ítems representa una conducta o forma de interacción por parte de la pareja que se considera abusiva (e.g., "Mi pareja me humilla", "Mi pareja me golpea o araña", "Mi pareja me grita continuamente"). Los estudios llevados a cabo con mujeres muestran adecuadas propiedades psicométricas con valores de fiabilidad de consistencia interna que oscilan entre .79 y .98 . Se ha usado de forma frecuente como criterio de validez externa (Torres et al., 2010) y muestra valores adecuados de validez convergente con constructos relacionados (Sierra, Monge, Santos-Iglesias, Bermúdez, y Salinas, 2011). Además, se han propuesto puntos de corte para detectar el abuso por parte de la pareja (Sierra, Monge et al., 2011; Torres et al., 2010).

El principal problema de esta escala es la diversidad de estructuras factoriales encontradas. Sierra, Monge et al. (2011) informan de siete estructuras diferentes que combinan sus ítems en dos factores (Abuso fisico y Abuso no físico; Hudson y McIntosh, 1981; Plazaola-Castaño, Ruiz-Pérez, Escribà-Agüir, Jiménez-Martín, y Hernández-Torres, 2009; Sierra, Ortega, Santos, y Gutiérrez, 2007; Tang, 1998; Torres et al., 2010) o en tres factores (Abuso fisico, Abuso no fisico y Control de conductas; Campbell, Campbell, King, Parker, y Ryan, 1994; Cook, Conrad, Bender, y Kaslow, 2003). Esta variabilidad se debe a que a cada una de estas estructuras se ha llegado usando muestras distintas y, en la mayoría de las ocasiones, a través de análisis factorial exploratorio, siendo muy pocos los casos en los que se ha tratado de confirmar las estructuras existentes. En este sentido, los trabajos llevados a cabo por Sierra y colaboradores (Sierra,
Costa, y Santos-Iglesias, 2010; Sierra, Monge et al., 2011; Sierra, Santos-Iglesias, y Gutiérrez-Quintanilla, 2010) han mostrado que el uso de análisis factorial confirmatorio es una estrategia más efectiva para el análisis de la estructura factorial del ISA, encontrándose de forma sistemática que en mujeres la estructura que mejor ajusta es la propuesta por Tang (1998), compuesta por 19 ítems agrupados en dos factores: Abuso físico y Abuso no físico.

El ISA ha sido empleado y adaptado en diversos países y muestras de mujeres (véase Sierra, Monge et al., 2011). Existe también evidencia de que ha sido empleado en muestras de varones con el objetivo de evaluar la frecuencia del abuso por parte de la pareja (Ernst, Houry, Nick, y Weiss, 1998; Ernst, Nick, Weiss, Houry, y Mills, 1997; Ernst, Weiss, Nick, Casalletto, y Garza, 2000); sin embargo, no se tiene conocimiento de que haya sido previamente adaptado y validado para su uso en muestras de varones. Por esta razón, el objetivo de este estudio es analizar algunas propiedades psicométricas del Index of Spouse Abuse en una muestra de varones españoles. En primer lugar se examinará la estructura factorial mediante análisis factorial confirmatorio, contrastando las siete estructuras citadas por Sierra, Monge et al. (2011). Las estructuras sometidas a contraste se pueden observar en la Tabla 1. Una vez confirmada la estructura se llevará a cabo un análisis de ítems y de fiabilidad de cada factor. Por último, se analizará la validez convergente de la escala; en este sentido, se hipotetiza que los varones con elevadas puntuaciones en abuso mostrarán bajas puntuaciones en asertividad sexual, ya que el abuso en la pareja inhibe las respuestas sexualmente asertivas (Apt y Hurlbert, 1993; Santos-Iglesias y Sierra, 2010a). Por último, se elaborará una clasificación de las conductas de abuso más frecuentes, con el objetivo de analizar el tipo de violencia de pareja sufrida por los varones de la muestra.

\section{Método}

\section{Participantes}

Los participantes fueron seleccionados de la población general mediante un muestreo incidental por cuotas, que incluía varones del norte y sur de España, y varones residentes en ciudades mayores y menores de 50.000 habitantes. Los criterios de inclusión fueron: ser mayor de edad, mantener una relación de pareja heterosexual de al menos 6 meses de duración y tener actividad sexual en esa relación de pareja.

Participaron un total de 598 varones españoles, cuya edad osciló entre 18 y 77 años $(M=40.20 ; D T=13.71)$. 
Tabla 1. Estructuras factoriales sometidas a contraste.

\begin{tabular}{|c|c|c|c|c|}
\hline \multirow[b]{2}{*}{ Autores } & \multirow[b]{2}{*}{ Número factores/ítems } & \multicolumn{3}{|c|}{ Factores } \\
\hline & & Abuso no fisico & Abuso físico & Control de conductas \\
\hline Hudson y McIntosh (1981) & $2 / 30$ & 19 ítems & 11 ítems & \\
\hline Campbell et al. (1994) & $3 / 30$ & 17 ítems & 7 ítems & 6 ítems \\
\hline Tang (1998) & $2 / 19$ & 12 ítems & 7 ítems & \\
\hline Cook et al. (2003) & $3 / 22$ & 11 ítems & 4 ítems & 7 ítems \\
\hline Sierra et al. (2007) & $2 / 22$ & 14 ítems & 8 ítems & \\
\hline Plazaola-Castaño et al. (2009) & $2 / 30$ & 22 ítems & 8 ítems & \\
\hline Torres et al. (2010) & $2 / 30$ & 23 ítems & 7 ítems & \\
\hline
\end{tabular}

El $2 \%(n=12)$ de los varones no tenía estudios, un $20.6 \%$ $(n=123)$ estudios primarios, el 33\% $(n=197)$ estudios secundarios y el 44.4\% $(n=265)$ estudios superiores. La mayoría de ellos son de religión cristiana (73\%), el 26,5\% no muestran preferencia por ninguna religión, mientras que sólo un $0.2 \%$ y un $0.3 \%$ informan ser practicantes de religión islámica y budista, respectivamente. Por último, el $0.5 \%(n=3)$ asisten a actos religiosos diariamente, el $4.5 \%(n=27)$ lo hace una vez por semana, el 7.7\% $(n=46)$ alguna vez al mes, el 50.3\% $(n=301)$ alguna vez al año y el $37 \%(n=221)$ nunca.

\section{Instrumentos}

Se empleó un cuestionario sociodemográfico en el que se recogía información sobre la edad, si mantenían una relación de pareja, si tenían actividad sexual dentro de esa relación, tamaño de la población de residencia, si residen en el norte o sur de España, nivel de estudios, religión y frecuencia de asistencia a actos religiosos.

Index of Spouse Abuse (Hudson y McIntosh, 1981). Se aplicó la traducción española de Cáceres (2002) compuesta por 30 ítems, que se responden en una escala tipo Likert de 0 (nunca) a 4 (casi siempre). Mayores puntuaciones indican una mayor frecuencia de abuso. Sus propiedades psicométricas han sido descritas anteriormente.

Sexual Assertiveness Scale (SAS; Morokoff et al., 1997). Se empleó la adaptación española de Sierra, VallejoMedina y Santos-Iglesias (2011) compuesta por 18 ítems que evalúan la habilidad para mostrar respuestas sexualmente asertivas en tres dominios: inicio de la actividad sexual, rechazo de la actividad sexual no deseada y uso de métodos anticonceptivos con la pareja para prevenir embarazos y enfermedades de transmisión sexual (ETS). Se responde en una escala tipo Likert de 0 (nunca) a 4 (siempre) y mayores puntuaciones indican mayor asertividad sexual. La adaptación española mostró una fiabilidad de consistencia interna superior a .75 en las tres dimensiones y adecuados índices de validez. Los coeficientes de fiabilidad alcanzados en la presente muestra fueron .60 para Inicio, .67 para Rechazo y .84 para Embarazo-Enfermedades de Transmisión Sexual.

\section{Procedimiento}

Los participantes fueron evaluados de forma individual o en grupos (en aulas o salas dispuestas para ello). Las evaluaciones se llevaron a cabo por un investigador entrenado para tal efecto que proporcionaba las mismas instrucciones a todos los participantes. Antes de solicitar su colaboración se les daba información sobre el objetivo de la investigación. Posteriormente se solicitaba su colaboración garantizándoles su anonimato y la confidencialidad de las respuestas ofrecidas, obteniendo el consentimiento verbal por parte de los interesados.

\section{Análisis de datos}

El análisis factorial confirmatorio se llevó a cabo mediante modelos de ecuaciones estructurales usando LISREL 8.51 (Jöreskog y Sörbom, 2001). Debido a la asimetría provocada por el efecto suelo en las respuestas al cuestionario (elevada frecuencia de respuestas en el 0) los datos fueron analizados tratándolos como si fueran categóricos, empleando el estimador Diagonally Weighted Least Squares (Brown, 2006). Para evaluar el ajuste del modelo se realizó una evaluación conjunta de una serie de indicadores (Tanaka, 1993). Se analizó el valor de $\chi^{2}$ y su significación asociada, sin embargo, es conocida su dependencia del tamaño muestral y de la ausencia de normalidad multivariada. Por ello, se emplearon además el Comparative Fit Index (CFI), el Tucker-Lewis Index (TLI) y el Root Mean Square Error of Approximation (RMSEA). Valores iguales o inferiores a 0.06 en RMSEA e iguales a 0.95 o superiores en CFI y TLI son indicadores de un buen ajuste (Hu y Bentler, 1999). 


\section{Resultados}

\section{Análisis factorial confirmatorio}

Los resultados del análisis factorial confirmatorio (véase la Tabla 2) muestran que el modelo con mejor ajuste es el compuesto por 3 factores y 30 ítems propuesto por Campbell et al. (1994), ya que el RMSEA puede ser considerado el mejor indicador de ajuste global (Marsh, Balla y Hau, 1996). La estructura factorial resultante puede verse en la Figura 1.

\section{Análisis de items y fiabilidad}

El análisis de ítems y fiabilidad de consistencia interna se llevó a cabo por separado para cada una de las subescalas obtenidas en el análisis factorial confirmatorio. En el caso de la escala Abuso no fisicico, en la Tabla 3 se puede observar que las medias oscilan entre 0.13 (ítem 28) y 1.73 (ítem 3). Las desviaciones típicas no son excesivamente bajas, excepto para el ítem $28(D T=0.55)$, lo que indica una buena variabilidad de puntuaciones en la escala de respuesta. Las correlaciones ítem-total corregidas superan todas el valor
.35 (Nunnally y Bernstein, 1995), a excepción de los ítems 3 y 14. Sin embargo, la eliminación de estos dos ítems no supone en ningún caso una mejora sustancial del valor de alfa de Cronbach, que fue igual a .81 .

En el caso de la escala Control de conductas (véase la Tabla 4), las medias oscilaron entre 0.43 del ítem 18 y 1.73 del ítem 6. Por su parte, las desviaciones típicas se hallaban en torno a la unidad, siendo la de los ítems 18 y 21 las más bajas de todas $(D T=0.85)$. Más problemáticas fueron las correlaciones ítem-total corregidas, ya que tres de los seis ítems muestran valores por debajo de .35 , concretamente los ítems 6, 19 y 21. Únicamente, la eliminación del ítem 21 mejora el valor alfa de Cronbach que fue para esta subescala igual a .60. Sin embargo, se decidió mantener este ítem para preservar la estructura original de Campbell et al. (1994).

Por último, la escala Abuso físico mostró medias y desviaciones típicas muy bajas (véase la Tabla 5); las medias oscilaron entre 0.05 (ítem 17) y 0.40 (ítem 7) y ninguna desviación típica superó la unidad. Las correlaciones ítem-total corregidas igualan o superan en todos los casos el valor .35. La eliminación de ítems no mejoraba el valor de fiabilidad (alfa de Cronbach) de la escala, que fue igual a .79.

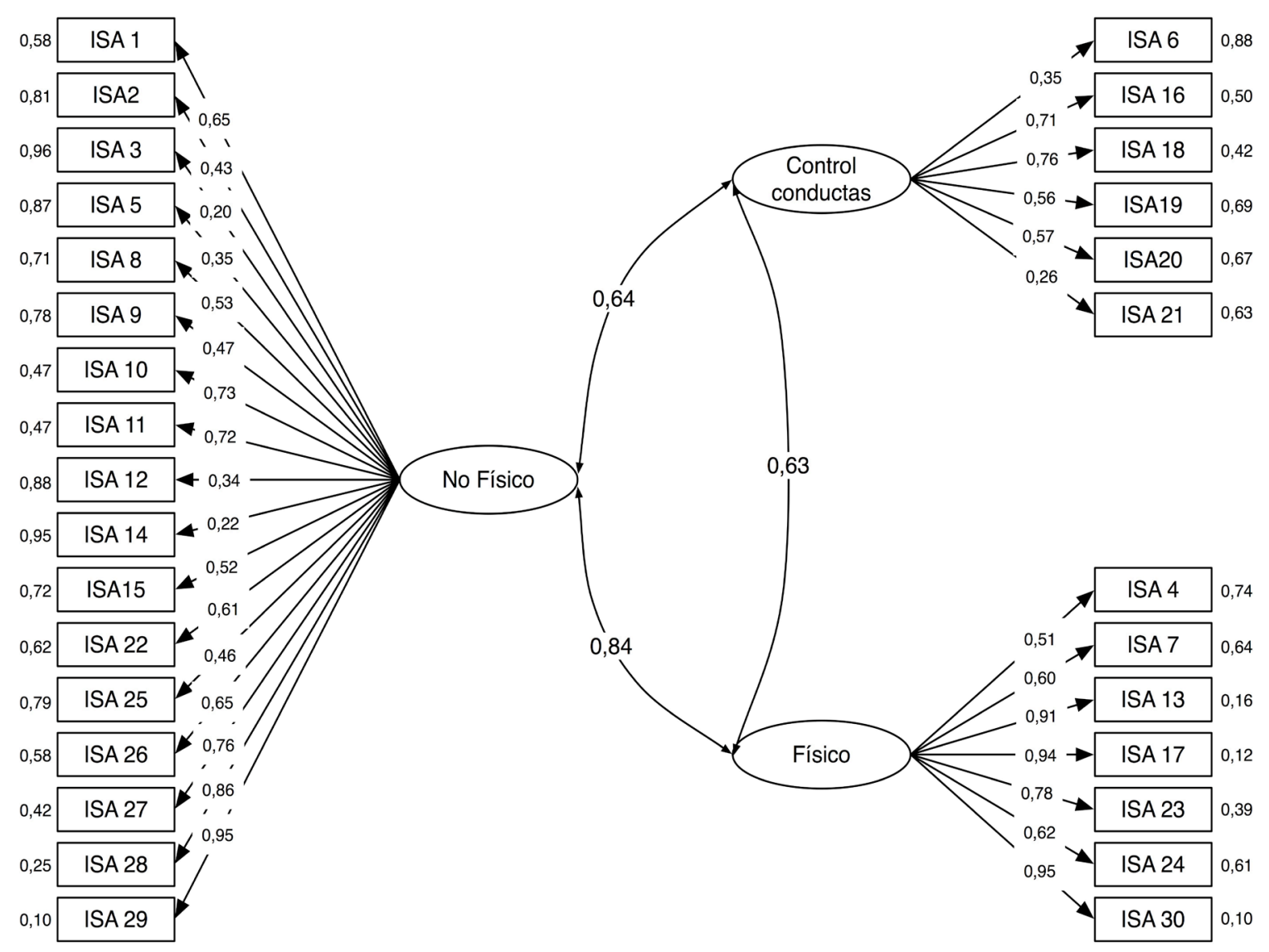

Figura 1. Modelo de 30 items y 3 factores resultante en la muestra de varones españoles. 
Tabla 2. Índices de ajuste de los modelos sometidos a contraste.

\begin{tabular}{lccccc}
\hline Modelos & $\chi^{2}$ & RMSEA & 90\% c.i. RMSEA & CFI & TLI \\
\hline Hudson y McIntosh (1981) & $923.72 * * *$ & 0.046 & $0.042-0.050$ & 0.98 & 0.98 \\
Campbell et al. (1994) & $640.66^{* * *}$ & 0.032 & $0.027-0.036$ & 0.98 & 0.98 \\
Tang (1998) & $261.52 * * *$ & 0.035 & $0.028-0.042$ & 0.98 & 0.98 \\
Cook et al. (2003) & $508.44 * * *$ & 0.050 & $0.044-0.055$ & 0.98 & 0.98 \\
Sierra et al. (2007) & $541.44 * * *$ & 0.044 & $0.039-0.050$ & 0.98 & 0.97 \\
Plazaola-Castaño et al. (2009) & $1.170 .95 * * *$ & 0.056 & $0.053-0.060$ & 0.98 & 0.97 \\
\hline
\end{tabular}

Nota. El modelo de Torres et al. (2010) no aparece en la tabla ya que la solución no convergió, por lo que no hay indicadores de ajuste. $* * * p<.001$

Tabla 3. Descriptivos de los items de la escala Abuso no físico.

\begin{tabular}{llccc}
\hline İtems & M & DT & ri-t & a-i \\
\hline 1. Me humilla & 0.44 & 0.72 & .49 & .79 \\
2. Me exige que obedezca a sus caprichos & 1.03 & 1.03 & .36 & .80 \\
3. Se irrita si le digo que come, bebe o fuma demasiado & 1.73 & 1.26 & .22 & .81 \\
5. Se enfada mucho si no hago lo que quiere cuando ella quiere & 1.52 & 1.11 & .38 & .80 \\
8. Me repite que soy poco atractivo & 0.53 & 0.86 & .39 & .80 \\
9. Me repite que no podría valerme sin ella & 0.67 & 1.03 & .37 & .80 \\
10. Se cree que soy su esclavo & 0.35 & 0.74 & .57 & .79 \\
11. Me insulta o avergüenza delante de otros & 0.44 & 0.73 & .54 & .79 \\
12. Se enfada mucho si me muestro en desacuerdo con sus puntos de vista & 1.70 & 1.00 & .36 & .80 \\
14. No me presta dinero & 0.67 & 1.14 & .07 & .82 \\
15. Me repite que soy tonto & 0.65 & 0.89 & .41 & .80 \\
22. Me grita continuamente & 0.85 & 0.98 & .55 & .79 \\
25. Está siempre dando órdenes & 1.41 & 1.15 & .44 & .80 \\
26. Me dice cosas que no se pueden aguantar & 0.83 & 0.94 & .53 & .79 \\
27. Actúa como una cobarde conmigo & 0.26 & 0.61 & .48 & .80 \\
28. Le tengo miedo & 0.13 & 0.55 & .55 & .79 \\
29. Me trata como si fuera basura & 0.22 & 0.63 & .67 & .79 \\
\hline
\end{tabular}

Nota: ri-t: correlación ítem-total corregida; $\alpha$-i: alfa de Cronbach si se elimina el ítem.

Tabla 4. Descriptivos de los items de la escala control de conductas.

\begin{tabular}{|c|c|c|c|c|}
\hline Ítems & $\mathrm{M}$ & DT & ri-t & $\mathrm{a}-\mathrm{i}$ \\
\hline 6. Se muestra celosa y suspicaz con mis amistades & 1.73 & 1.26 & .29 & .58 \\
\hline 16. Me obliga a quedarme en casa si no tengo nada que hacer por ahí & 0.60 & 0.99 & .41 & .52 \\
\hline 18. Cree que no debería salir de casa o asistir a reuniones, cursos.... & 0.43 & 0.85 & .46 & .51 \\
\hline 19. No es amable conmigo & 0.78 & 1.02 & .23 & .59 \\
\hline 20. No quiere que salga con mis amistades & 1.03 & 1.09 & .55 & .45 \\
\hline 21. Me exige relaciones sexuales. aunque esté cansado & 0.68 & 0.85 & .11 & .63 \\
\hline
\end{tabular}

Nota: ri-t: correlación ítem-total corregida; $\alpha$-i: alfa de Cronbach si se elimina el ítem. 
Tabla 5. Descriptivos de los items de la escala abuso físico.

\begin{tabular}{lllll}
\hline Ítems & M & DT & ri-t & a-i \\
\hline 4. Me fuerza a hacer actos sexuales que no me gustan & 0.30 & 0.65 & .35 & .79 \\
7. Me golpea o araña & 0.40 & 0.77 & .46 & .78 \\
13. Me amenaza con un arma o cuchillo & 0.09 & 0.47 & .69 & .73 \\
17. Me ha llegado a golpear tan fuerte que llegué a necesitar asistencia médica & 0.05 & 0.35 & .62 & .75 \\
23. Me da golpes en la cara y en la cabeza & 0.13 & 0.46 & .58 & .74 \\
24. Se vuelve agresiva cuando bebe & 0.20 & 0.61 & .40 & .78 \\
30. Actúa como si fuera a matarme & 0.09 & 0.46 & .75 & .72 \\
\hline
\end{tabular}

Nota: ri-t: correlación ítem-total corregida; $\alpha$-i: alfa de Cronbach si se elimina el ítem.

Tabla 6. Clasificación de las conductas de abuso más frecuentes en la muestra de varones.

\begin{tabular}{|c|c|c|}
\hline Ítem & \% víctimas & Dimensión \\
\hline 12. Se enfada mucho si me muestro en desacuerdo con sus puntos de vista & 90.3 & ANF \\
\hline 5. Se enfada mucho si no hago lo que quiere cuando ella quiere & 82.1 & ANF \\
\hline 6. Se muestra celosa y suspicaz con mis amistades & 80.4 & $\mathrm{CC}$ \\
\hline 3. Se irrita si le digo que come. bebe o fuma demasiado & 78.3 & ANF \\
\hline 25. Está siempre dando órdenes & 76.8 & ANF \\
\hline 2. Me exige que obedezca a sus caprichos & 63.2 & ANF \\
\hline 20. No quiere que salga con mis amistades & 60.7 & $\mathrm{CC}$ \\
\hline 26. Me dice cosas que no se pueden aguantar & 55.9 & ANF \\
\hline 22. Me grita continuamente & 55 & ANF \\
\hline 19. No es amable conmigo & 49.3 & $\mathrm{CC}$ \\
\hline 21. Me exige relaciones sexuales. aunque esté cansado & 48.8 & $\mathrm{CC}$ \\
\hline 15. Me repite que soy tonto & 44.5 & ANF \\
\hline 9. Me repite que no podría valerme sin ella & 39.5 & ANF \\
\hline 16. Me obliga a quedarme en casa si no tengo nada que hacer por ahí & 34.8 & $\mathrm{CC}$ \\
\hline 8. Me repite que soy poco atractivo & 34.3 & ANF \\
\hline 1. Me humilla & 33.6 & ANF \\
\hline 14. No me presta dinero & 33.6 & ANF \\
\hline 11. Me insulta o avergüenza delante de otros & 32.8 & ANF \\
\hline 7. Me golpea o araña & 27.4 & $\mathrm{AF}$ \\
\hline 18. Cree que no debería salir de casa o asistir a reuniones, cursos.... & 26.6 & $\mathrm{CC}$ \\
\hline 10. Se cree que soy su esclavo & 23.2 & ANF \\
\hline 4. Me fuerza a hacer actos sexuales que no me gustan & 22.7 & $\mathrm{AF}$ \\
\hline 27. Actúa como una cobarde conmigo & 18.6 & ANF \\
\hline 29. Me trata como si fuera basura & 13.9 & ANF \\
\hline 24. Se vuelve agresiva cuando bebe & 12.2 & $\mathrm{AF}$ \\
\hline 28. Le tengo miedo & 10.7 & ANF \\
\hline 23. Me da golpes en la cara y en la cabeza & 8.9 & $\mathrm{AF}$ \\
\hline 30. Actúa como si fuera a matarme & 4.7 & $\mathrm{AF}$ \\
\hline 13. Me amenaza con un arma o cuchillo & 4.5 & $\mathrm{AF}$ \\
\hline 17. Me ha llegado a golpear tan fuerte que llegué a necesitar asistencia médica & 3 & $\mathrm{AF}$ \\
\hline
\end{tabular}

Nota: ANF: Abuso no físico; CC: Control de conductas; AF: Abuso físico.

\section{Análisis de validez}

Los análisis de validez convergente mostraron correlaciones significativas de signo negativo entre el abuso no físico y la asertividad de inicio $(r=-.08, p=.04)$ y la asertividad de rechazo $(r=-.13, p=.002)$. El control de conductas se relacionó con la asertividad para el uso de métodos anticonceptivos $(r=-.15, p<.001)$.

\section{Clasificación de las conductas de abuso más frecuentes}

Los resultados de la Tabla 6 muestran que las conductas de abuso más frecuentes son, sobre todo, de abuso no físico, ya que entre las cinco primeras, cuatro corresponden a esa categoría. Así la primera es "se enfada mucho conmigo si me muestro en desacuerdo con sus puntos de vista", seguida 
por "Se enfada mucho si no hago lo que quiere cuando ella quiere". En tercer lugar encontramos una conducta de control ("Se muestra celosa y suspicaz con mis amistades").

Las conductas pertenecientes a la subescala Control de conductas son bastante frecuentes, pues la mayoría se encuentran en la primera mitad de la clasificación. Por el contrario, las conductas menos frecuentes son las de abuso físico, ya que cuatro de ellas ocupan los últimos cuatro puestos (siendo la última "Me ha llegado a golpear tan fuerte que llegué a necesitar asistencia médica”) y ninguna ocupa la primera mitad de la clasificación.

\section{Discusión}

Debido a la necesidad de contar con instrumentos de evaluación para detectar el abuso por parte de la pareja (Kapur y Windish, 2011) y a su escasez para evaluar el abuso en hombres (Hays y Emelianchik, 2009; Thompson, et al., 2006) se ha plantado este estudio cuyo objetivo fue analizar algunas propiedades psicométricas del Index of Spouse Abuse (Hudson y McIntosh, 1981) en una muestra de varones españoles. Los resultados muestran una versión compuesta por 30 ítems agrupados en tres factores (Abuso físico, Control de conductas y Abuso físico) con buenos índices de fiabilidad y validez y, por tanto, avalan un instrumento adecuado para la evaluación del abuso de pareja en varones.

El resultado del análisis factorial confirmatorio demuestra, al igual que sucedió en ocasiones previas (Sierra, Costa et al., 2010; Sierra, Monge et al., 2011; Sierra, Santos-Iglesias et al., 2010), que es posible determinar la estructura factorial del instrumento tratando de confirmar alguna de las estructuras previamente analizadas. Esto supone una ventaja frente al uso del análisis factorial exploratorio que se ha usado en trabajos previos (Plazaola-Castaño et al., 2009; Sierra et al., 2007; Torres et al., 2010), ya que permite obtener una estructura factorial consistente con alguna de las que ya existen, en lugar de ofrecer una estructura nueva y que, en muchas ocasiones, no concuerda con las anteriores. De esta manera no aumentan el número de estructuras factoriales disponibles, ya que, recordemos, existen dos estructuras diferentes en España (Plazaola-Castaño et al., 2009; Torres et al., 2010) y otras dos en muestras de mujeres salvadoreñas (Sierra et al., 2007; Sierra, Santos-Iglesias et al., 2010).

Además de este resultado de carácter general, se observa como en el caso de los hombres la estructura que mejor ajusta es la de tres factores que incluye, además de los componentes de abuso físico y no físico, un componente de control de conductas. Esto puede ser debido a que los hombres sufren más este tipo de abusos frente a otros menos frecuentes en su caso como el abuso físico (Holtzworth-Munroe, 2005) $\mathrm{y}$, por tanto, los hombres son más sensibles a este tipo de conductas. De hecho, se observa en la clasificación que la mayoría de las conductas de abuso de la escala Control de conductas se encuentran en la primera mitad de la tabla.

Los resultados del análisis de ítems y del análisis de fiabilidad muestran, de forma general, adecuadas propiedades de los ítems. Sin embargo, un examen detallado muestra que las medias y desviaciones típicas de los distintos ítems que componen las diferentes subescalas son, en líneas generales, muy bajas, especialmente en el caso de los de abuso físico. Esto se debe al hecho de que se evalúan conductas poco frecuentes en las que es fácil encontrar un efecto suelo en las respuestas, donde la mayoría de los participantes responden en el rango más bajo de la escala. Por tanto, a pesar de que es deseable que las medias de los ítems se encuentren en el punto medio de la escala de respuesta y las desviaciones típicas sean superiores a uno (Carretero-Dios y Pérez, 2005), en el caso del constructo a evaluar es comprensible el patrón de respuestas obtenido, más aún cuando la muestra ha sido recogida de la población general. Respecto a las correlaciones ítem-total corregidas, la mayoría de los ítems no presentan problemas, salvo contadas excepciones. Por ejemplo, el ítem de la escala Abuso no físico "no me presta dinero" parece tener poco que ver con otros como "Me humilla" o "Me repite que soy tonto". De la misma forma, el ítem "No es amable conmigo" de la escala Control de conductas parece no estar muy relacionado con otros como "Se muestra celosa y suspicaz con mis amistades". Sin embargo, esos ítems no fueron eliminados por dos razones. En primer lugar, su eliminación no contribuía de forma significativa a aumentar el valor alfa de Cronbach de sus respectivas escalas. En segundo lugar, su eliminación supone una alteración de la estructura propuesta por Campbell et al. (1994) y además reduce el número de conductas de abuso que pueden explorarse con esta estructura, reduciendo la capacidad de la escala para detectar casos de violencia de pareja. Por último, los indicadores de fiabilidad son adecuados en todas las subescalas, aunque la consistencia interna de la escala Control de conductas es algo baja, lo cual podría deberse a un menor número de ítems.

Respecto a los indicadores de validez, se encuentra que el abuso no físico dificulta las respuestas asertivas para el inicio de la actividad sexual y para el rechazo de la actividad sexual no deseada. Este resultado es consistente con la literatura previa que muestra que las mujeres que sufren abuso por parte de la pareja muestran también menos asertividad 
sexual (Apt y Hurlbert, 1993). Además, es necesario recordar que el instrumento de evaluación de la asertividad sexual empleado por Apt y Hurlbert (1993) fue el Hurlbert Index of Sexual Assertiveness, que está compuesto por ítems que evalúan tanto inicio como rechazo de la actividad sexual (Santos-Iglesias y Sierra, 2010b), exactamente las dos dimensiones en las que se encuentra la relación en el presente estudio. Este resultado es significativo, además, porque existen estudios previos que demuestran la asociación entre el abuso no físico y la ausencia de asertividad sexual (Santos Iglesias, Sierra, y Vallejo-Medina, 2012). Por último, también se encuentra una relación negativa entre el control de conductas y la falta de asertividad para el uso de métodos anticonceptivos. Esto podría parecer lógico, ya que al igual que se controlan conductas como salir de casa o ver a los amigos, puede que de la misma manera se controle el uso del preservativo.

Para finalizar hay que señalar que, tal y como indican estudios previos, a pesar de que el varón puede sufrir abuso por parte de la pareja con la misma frecuencia que la mujer (Archer, 2002; Randle y Graham, 2011), éstos suelen ser víctimas de formas menos severas de abuso (HoltzworthMunroe, 2005). Así, se ha mostrado que las conductas más frecuentes tienen que ver con abuso no físico y control de conductas como enfadarse con la pareja o mostrarse celoso, mientras que las menos frecuentes son las de abuso físico como recibir golpes o amenazas. Sería interesante, no obstante, realizar un estudio para ver no sólo si el orden de estas conductas es el mismo en hombres y mujeres españoles, sino también las diferencias en las frecuencias entre ambos.

A modo de resumen se puede concluir que la adaptación española del Index of Spouse Abuse para su uso en varones ofrece un instrumento con 30 ítems que evalúan abuso físico, abuso no físico y control de conductas. El instrumento muestra adecuadas garantías psicométricas para su uso en muestras de varones españoles, sin embargo hacen falta más estudios para garantizar estas propiedades y otras no estudiadas. Así, sería útil disponer de indicadores de validez discriminante o puntos de corte para la detección de abuso, así como una versión abreviada que pueda ser empleada para realizar screening en ámbitos aplicados (Feldhaus et al., 1997; Kapur y Windish, 2011). Además, hay que señalar que el estudio se ha llevado a cabo con una muestra no representativa de los varones españoles, lo que impide generalizar los resultados y obliga a realizar más estudios que confirmen las propiedades de esta escala.

\section{Referencias}

Apt, C., y Hurlbert, D. F. (1993). The sexuality of women in physically abusive marriages: A comparative study. Journal of Family Violence, 8, 57-69.

Archer, J. (2002). Sex differences in physically aggressive acts between heterosexual partners: A meta-analytic review. Aggression and Violent Behavior, 7, 313-351.

Brown, T. A. (2006). Confirmatory factor analysis for applied research. Nueva York, NY: The Guilford Press.

Cáceres, J. (2002). Análisis cuantitativo y cualitativo de la violencia doméstica en la pareja. Cuadernos de Medicina Psicosomática y Psiquiatría de Enlace, 61, 57-67.

Campbell, D. W., Campbell, J. C., King, C., Parker, B., y Ryan, J. (1994). The reliability and factor structure of the Index of Spouse Abuse with African-American women. Violence and Victims, 9, 259-274.

Carney, M., Buttell, F., y Dutton, D. (2007). Women who perpetrate intimate partner violence: A review of the literature with recommendations for treatment. Aggression and Violent Behavior, 12, 108-115.

Carretero-Dios, H., y Pérez, C. (2005). Normas para el desarrollo y revisión de estudios instrumentales. International Journal of Clinical and Health Psychology, 5, 521-551.

Cook, S. L., Conrad, L., Bender, M., y Kaslow, N. J. (2003). The internal validity of the Index of Spouse Abuse in African American women. Violence and Victims, 18, 641-657.

Ernst, A. A., Houry, D., Nick, T. G., y Weiss, S. J. (1998). Domestic violece awareness and prevalence in a first-year medical school class. Academic Emergency Medicine, 5, 64-68.

Ernst, A. A., Nick, T. G., Weiss, S. J., Houry, D., y Mills, T. (1997). Domestic violence in an inner-city ED. Annals of Emergency Medicine, 30, 190-198.

Ernst, A. A., Weiss, S. J., Nick, T. G., Casalletto, J., y Garza, A. (2000). Domestic violence in a university emergency department. Southern Medical Journal, 93, 176-181.

Feldhaus, K. M., Koziol-McLain, J., Amsbury, H. L., Norton, I. M., Lowenstein, S. R., y Abbott, J. T. (1997). Accuracy of 3 brief screening questions for detecting partner violence in the emergency department. JAMA, 277, 1357-1361.

Goldenson, J., Spidel, A., Greaves, C., y Dutton, D. (2009). Female perpetrators of intimate partner violence: within-group heterogeneity, related psychopathology, and a review of current treatment with recommendations for future. Journal of Aggression, Maltreatment $y$ Trauma, 18, 752-769.

Hays, D. G., y Emelianchik, K. (2009). A content analysis of intimate partner violence assessments. Measurement Evaluation in Counseling and Development, 42, 139-153.

Holtzworth-Munroe, A. (2005). Male versus female intimate partner violence: Putting controversial findings into context. Journal of Marriage and the Family, 67, 1120-1125.

Hu, L., y Bentler, P. M. (1999). Cutoff criteria for fit indexes in covariance structure analysis: Conventional criteria versus new alternatives. Structural Equation Modeling, 6, 1-55.

Hudson, W. W., y McIntosh, S. R. (1981). The assessment of spouse abuse: Two quantifiable dimensions. Journal of Marriage and the Family, 43, 873-888.

Ibaceta, F. (2011). Violencia en la pareja: ¿es posible la terapia conjunta? Terapia Pisocológica, 29, 117-125.

Jöreskog, K. G., y Sörbom, D. (2001). LISREL 8.51. Chicago, IL: Scientific Software International.

Kapur, N. A., y Windish, D. M. (2011). Optimal methods to screening men and women for intimate partner violence: Results from an internal medicine residency continuity clinic. Journal of Interpersonal Violence, 26, 2335-2352. 
Loinaz, I., Echeburua, E., y Ullate, M. (2012). Estilo de apego, empatia y autoestima en agresores de pareja. Terapia Psicológica, 30, 61-70.

Loinaz, I., Ortiz-Tallo, M., y Ferragut, M. (2012). MCMI-III Grossman personality facets among partner-violence men in prison. International Journal of Clinical and Health Psycghology, 12, 389-404.

Marsh, H. W., Balla, J. R., y Hau, K. T. (1996). An evaluation of incremental indexes: A clarification of mathematical and empirical properties. In G. A. Marcoulides y R. E. Schumacker (Eds.), Advanced structural equation modeling techniques (pp. 315-353). Mahwah, NJ: Lawrence Erlbaum.

Morokoff, P. J., Quina, K., Harlow, L. L., Whitmire, L., Grimley, D. M., Gibson, P. R., y Burkholder, G. J. (1997). Sexual Assertiveness Scale (SAS) for women: Development and validation. Journal of Personality and Social Psychology, 73, 790-804.

Novo, M., Fariña, F., Seijo, M.D., y Arce, R. (2012). Assessment of a community rehabilitation programme in convicted male intimatepartner violence offeders. International Journal of Clinical and Health Psychology, 12, 219-234.

Nunnally, J. C., y Bernstein, I. J. (1995). Teoría psicométrica. México, DF.: McGraw Hill.

Plazaola-Castaño, J., Ruiz-Pérez, I., Escribà-Agüir, V., Jiménez-Martín, J. M., y Hernández-Torres, E. (2009). Validation of the Spanish version of the index of Spouse Abuse. Journal of Women's Health, 18, 499-506.

Póo, A. M., y Vizcarra, M. B. (2011). Diseño, implementación y evaluación de un programa de prevención de la violencia en el noviazgo. Terapia Psicológica, 29, 213-223.

Randle, A. A., y Graham, C. A. (2011). A review of the evidence on the effects of intimate partner violence on men. Psychology of Men y Masculinity, 12, 97-111.

Rodríguez-Franco, L., López-Cepero Borrego, J., Rodríugez-Díaz, F.J., Bringas Molleda, C., Estrada Pineda, C., Antuña Bellerín M.A., y Quevedo-Blasco, R. (2012). Labeling dating abuse: Undectected abuse among Spanish adolescents and young adults. International Journal of Clinical and Health Psychology, 12, 55-67.

Saldivia, C., y Vizcarra, B. (2012). Consumo de drogas y violencia en el noviazgo en estudiantes universitarios del Sur de Chile. Terapia Psicológica, 30, 43-49.

Santos Iglesias, P., Sierra, J. C., y Vallejo-Medina, P. (2012). Predictors of sexual assertiveness: The role of sexual desire, arousal, attitudes, and partner abuse. Archives of Sexual Behavior. DOI: 10.1007/s10508012-9998-3

Santos-Iglesias, P., y Sierra, J. C. (2010a). El papel de la asertividad sexual en la sexualidad humana: una revisión sistemática. International Journal of Clinical and Health Psychology, 10, 553-577.

Santos-Iglesias, P., y Sierra, J. C. (2010b). Hurlbert Index of Sexual Assertiveness: A study of psychometric properties in a Spanish sample. Psychological Reports, 107, 39-57.

Sierra, J. C., Costa, N., y Santos-Iglesias, P. (2010). Un estudio acerca de las propiedades psicométricas de la versión brasileña del Index of Spouse Abuse. Revista Iberoamericana de Diagnóstico y Evaluación Psicológica, 30, 57-70.

Sierra, J. C., Monge, F. S., Santos-Iglesias, P., Bermúdez, M. P., y Salinas, J. M. (2011). Validation of a reduced Spanish version of the Index of Spouse Abuse. International Journal of Clinical and Health Psycho$\log y, 11,363-383$.

Sierra, J. C., Ortega, V., Santos, P., y Gutiérrez, J. R. (2007). Estructura factorial, consistencia interna e indicadores de validez de la versión española del Index of Spouse Abuse. Boletin de Psicología, 91, 83-96.

Sierra, J. C., Santos-Iglesias, P., y Gutiérrez-Quintanilla, J. R. (2010). Validación del Índice de Abuso en la Pareja en mujeres de El Salvador. Revista Mexicana de Psicología, 27, 5-14.

Sierra, J. C., Vallejo-Medina, P., y Santos-Iglesias, P. (2011). Propiedades psicométricas de la versión española de la Sexual Assertiveness Scale (SAS). Anales de Psicología, 27, 17-26.

Tanaka, J. S. (1993). Multifaceted conceptions of fit in structural models. En K. A. Bollen y J. S. Long (Eds.), Testing Structural Equation Models (pp. 10-39). Thousand Oaks, CA: Sage.

Tang, C. S. K. (1998). Psychological abuse of Chinese wives. Journal of Family Violence, 13, 299-314.

Thompson, M. P., Basile, K. C., Hertz, M. F., y Sitterle, D. (2006). Measuring intimate partner violence victimization and perpetration: A compendium of assessment tools. Atlanta, GA: Centers for Disease Control and Prevention, National Center for Injury Prevention and Control.

Torres, A., Navarro, P., García-Esteve, L., Tarragona, M. J., Ascaso, C., Herreras, Z., Gelabert, E., Imaz, M. L., Roca, A., Subirà, S., y MatínSantos, R. (2010). Detecting domestic violence: Spanish external validation of the Index of Spouse Abuse. Journal of Family Violence, $25,275-286$. 
